\title{
Long-term treatment with deferiprone enhances left ventricular ejection function when compared to deferoxamine in patients with thalassemia major
}

\author{
Aldo Filosa ${ }^{\mathrm{a}, *}$, Angela Vitrano b, Paolo Rigano ${ }^{\mathrm{c}}$, Giuseppina Calvaruso ${ }^{\mathrm{c}}$, Rita Barone ${ }^{\mathrm{c}}$, Marcello Capra ${ }^{\mathrm{d}}$, \\ Liana Cuccia ${ }^{d}$, Francesco Gagliardotto ${ }^{d}$, Lorella Pitrolo ${ }^{c}$, Luciano Prossomariti a, Maddalena Casale ${ }^{\text {a }}$, \\ Vincenzo Caruso ${ }^{\text {, }}$, Calogera Gerardi ${ }^{\mathrm{f}}$, Saveria Campisi ${ }^{\mathrm{g}}$, Paolo Cianciulli ${ }^{\mathrm{h}}$, Michele Rizzo ${ }^{\mathrm{i}}$, \\ Giuseppe D'Ascola $^{\mathrm{j}}$, Angela Ciancio ${ }^{\mathrm{k}}$, Aurelio Maggio ${ }^{\mathrm{c}}$ \\ a U.O.D. Centro per le Microcitemie, A.O.R.N. “A. Cardarelli," Napoli, Italy \\ b Dipartimento di Scienze Economiche, Aziendali e Statistiche, University of Palermo, Italy \\ c U.O.C. Ematologia II con Talassemia, A.O.R. "Villa Sofia-V. Cervello," Palermo, Italy \\ d U.O.C. Centro di Prevenzione Diagnosi e Cura della Talassemia, ARNAS Civico, Di Cristina, Benfratelli, Palermo, Italy \\ e U.O.D. Talassemia, ARNAS Garibaldi, Catania, Italy \\ ${ }^{\mathrm{f}}$ U.O.S. Talassemia, A.O.O.C.R. Sciacca, Italy \\ ${ }^{g}$ U.O.S. Centro Microcitemia, A.O. Umberto I, Siracusa, Italy \\ ${ }^{\text {h }}$ U.O. Talassemia, A.O.D. S. Eugenio, Roma, Italy \\ ${ }^{\text {i }}$ U.O.C. Ematologia, A.O. S. Elia, Caltanissetta, Italy \\ ${ }^{j}$ Centro Microcitemia, A.O. B.M.M., Reggio Calabria, Italy \\ k Ospedale Civile Madonna delle Grazie, U.O. Talassemia, Matera, Italy
}

\section{A R T I C L E I N F O}

\section{Article history:}

Submitted 16 February 2013

Revised 10 March 2013

Available online $\mathrm{xxxx}$

(Communicated by G. Stamatoyannopoulos, M.D., Dr. Sci., 11 March 2013)

\section{Keywords:}

Thalassemia major

Left ventricular ejection fraction (LVEF)

Deferiprone

Deferoxamine

Echocardiography

Chelation

\begin{abstract}
A B S T R A C T
Transfusion and iron chelation treatment have significantly reduced morbidity and improved survival of patients with thalassemia major. However, cardiac disease continues to be the most common cause of death. We report the left-ventricular ejection fraction, determined by echocardiography, in one hundred sixty-eight patients with thalassemia major followed for at least 5 years who received continuous monotherapy with deferoxamine $(N=108)$ or deferiprone $(N=60)$. The statistical analysis, using the generalized estimating equations model, indicated that the group treated with deferiprone had a significantly better left-ventricular ejection fraction than did those treated with deferoxamine (coefficient $0.97 ; 95 \% \mathrm{CI} 0.37 ; 1.6, p=0.002$ ). The heart may be particularly sensitive to iron-induced mitochondrial damage because of the large number of mitochondria and its low level of antioxidants. Deferiprone, because of its lower molecular weight, might cross into heart mitochondria more efficiently, improving their activity and, thereby, myocardial cell function. Our findings indicate that the long-term administration of deferiprone significantly enhances left-ventricular function over time in comparison with deferoxamine treatment. However, because of limitations related to the design of this study, these findings should be confirmed in a prospective, randomized clinical trial.
\end{abstract}

(c) 2013 Elsevier Inc. All rights reserved.

\section{Introduction}

Thalassemia is one of the most common genetic diseases worldwide with at least 60,000 severely affected subjects being born every year [1]. Iron overload is a major concern in these patients because of the need for red cell transfusions. Transfusion and iron chelation treatment have significantly improved survival and reduced morbidity in thalassemia major during the last three decades [2,3].

The effectiveness of chelation therapy has improved as a result of the introduction of the oral iron chelators, deferiprone (DFP) and deferasirox

* Corresponding author at: U.O.D. "Centro per le Microcitemie” A.O.R.N. “A. Cardarelli," Via A. Cardarelli, 980131 Napoli, Italy. Fax: + 390817472704

E-mail address: aldo.filosa@aocardarelli.it (A. Filosa).
(DFX). Each chelator can be given as monotherapy. DFP and deferoxamine (DFO) have been widely used in combination.

The detection and management of cardiac damage have improved over the last 10 years because of cardiac magnetic resonance-T2* (CMR-T2*) imaging, a non-invasive tool with which to monitor cardiac function associated with iron body burden [4,5]. Cardiac disease continues to be the most common cause of death in patients with thalassemia major $[3,6,7]$. Chelation therapy is aimed at improving myocardial function, as judged by the left ventricular ejection fraction (LVEF).

A multicenter, randomized, open-label, long-term, study of sequential DFP-DFO compared to DFP has been reported in patients with thalassemia major to assess LVEF [8]. Improvement in the LVEF after 1-year of DFP treatment versus DFO was even found $[9,10]$. However, the effect 
of DFP versus DFO on LVEF after long-term treatment has not so far been reported to our knowledge.

The aim of this study was to show if there was any difference in myocardial function as determined by 2-D echocardiography LVEF assessment when DFP and DFO treatment of thalassemia major patients was compared over a longer time period.

\section{Materials and methods}

\section{Patients and treatment}

This was a retrospective study of 168 patients with thalassemia major. Baseline clinical findings are shown in Table 1. All patients received at least 4 years of continuous monotherapy with DFO or DFP chelation treatment between September 30, 2002 and September 30, 2008. All enrolled subjects had echocardiographic study for LVEF. Among these, 108 received DFO treatment whereas 60 received DFP treatment (Table 1). DFO (Biofutura Pharma, Pomezia, Italy) was administered at $50 \mathrm{mg} / \mathrm{kg}$ per day by subcutaneous infusion (8-12 h) for 7 days. DFP (Apotex, Toronto, ON, Canada) was administered at $75 \mathrm{mg} / \mathrm{kg}$, divided into three oral daily doses, for 7 days a week. Chelation treatment was stopped or dose was adjusted in case of occurrence of severe adverse events (SAEs). Compliance was assessed by counting the pills in each returned bag of DFP and by assessing the number of infusions of DFO registered on the electronic pump (CronoTM, Gene S.r.l., Italy).

\section{Methods}

LVEF was defined as the fraction of end-diastolic volume that is ejected by the left ventricle in each beat. It was measured by a single operator with two-dimensional (2-D) echocardiography assessments dividing the stroke volume by the end-diastolic volume in each patient (Vivid S5, Gems Ultrasound, Tirat Carmel, Israel). Two-dimensional echocardiography was used to determine the LVEF and volumes (single plane area-length method), and to calculate right ventricular end-diastolic and end-systolic areas by planometry from the four-chamber view, according to previous recommendations [11]. The diagnosis of heart disease was based on standard clinical and instrumental findings including echocardiography and electrocardiogram [12]. Liver iron content (LIC) was determined on liver biopsies with an atomic spectrophotometer. Liver biopsy was performed only in patients on interferon treatment for chronic C hepatitis [13].

\section{Statistical models}

The generalized estimating equations (GEE) model was used [14] to evaluate if there was a statistically significant variation of LVEF over

Table 1

Baseline findings in the 168 patients included in this retrospective cohort study.

\begin{tabular}{llll}
\hline Findings & DFO-group & DFP-group & $p$-Value \\
\hline No. pts (168) & 108 & 60 & \\
Females no. (\%) & $55(51)$ & $22(37)$ & 0.25 \\
Age in years & $32 \pm 8.0$ & $31 \pm 7.0$ & 0.26 \\
Hemoglobin, g/L* & $9 \pm 0.83$ & $9 \pm 0.87$ & 0.91 \\
ALT, IU/L* & $53 \pm 39$ & $50 \pm 38$ & 0.71 \\
LIC, g/g per dw & $3.3 \pm 3.8$ & $2.9 \pm 2$ & 0.61 \\
Total transfusion, L/kg per year & $9 \pm 3$ & $8.7 \pm 2.1$ & 0.51 \\
Mean ferritin, mg/L & $1.5 \pm 1.1$ & $1.7 \pm 0.84$ & 0.37 \\
Mean basal EF <55\% (no.) & $45 \pm 8.0(26)$ & $52 \pm 2.0(6)$ & 0.05 \\
Basal mean ejection fraction & $58 \pm 10$ & $59 \pm 4$ & 0.23 \\
Mean age at DFO starting, years & $6 \pm 6$ & $6 \pm 4$ & 0.99 \\
Splenectomy no. (\%) & $59(60)$ & $26(44)$ & 0.18 \\
Cirrhosis no. (\%) & $21(20)$ & $5(8.0)$ & 0.01 \\
Arrhythmia no. (\%) & $16(15)$ & $11(18)$ & 0.81 \\
HCV-RNA positive no. (\%) & $40(42)$ & $18(30)$ & 0.38 \\
\hline
\end{tabular}

ALT, alanine aminotransferase; dw, dry weight; EF, ejection fraction; HCV, hepatitis C virus; IU, international units; LIC, liver iron content. time (four consecutive years) in the DFP versus DFO treated-groups and if this variation in the LVEF was statistically different between the two treated groups. LVEF values consisted of repeated observations over time on the same patient. These measurements, taken on the same patient, may not be independent one from another. One appropriate model, taking into account for the issue that repeated measurements might be correlated, is the generalized estimating equations (GEE) model [14]. This model includes the treatment-effect (treatment), the time-effect (time) and the treatment-by-time interaction effect (treatment $\times$ time) of the considered variables. This approach was implemented using the "xtgee" procedure in the Stata 11 software (Stata Corporation, College Station, TX, USA). These findings are reported on Table 2 and Fig. 1. Moreover, yearly LVEF\%, in both chelation groups divided by $<55$ and $>55$, was reported in Table 3 . Patient baseline clinical findings were reported with means and standard deviations (SD) for continuous variables and as proportions for dichotomous variables. All of the statistical analyses were performed under code at the Dipartimento di Scienze Economiche, Aziendali e Statistiche, University of Palermo (Italy).

\section{Results}

All patients were over 13 years of age (Table 1 ). The prevalence of males was higher in the DFP treatment group (Table 1). Among the 168 patients, 108 received DFO and 60 received DFP chelation treatment (Table 1). Baseline hematological, cardiac, and body iron burden findings, evaluated as serum ferritin level, LIC, total number of blood transfusions, arrhythmia, HCV-RNA positivity, and mean age at the start of chelation treatment were not significantly different between the two treated groups (Table 1). Presence of cirrhosis showed a statistically significant difference, at baseline, between DFP versus DFO group (Table 1). Compliance was $92 \pm 7 \%$ (range 37-100\%) and $70 \pm 6 \%$ (range $25-100 \%$ ) in the DFP versus the DFO treatment groups, respectively. The serum ferritin levels at the end of the study were $1.4 \pm 0.96$ versus $1.6 \pm 0.91 \mathrm{mg} / \mathrm{L}(p<0.01)$ in DFO compared to DFP, respectively. The pre-transfusional $\mathrm{Hb}$ level was not statistically different between the two chelation groups ( $t=1.6, p=0.12)$, excluding this finding as a possible bias of the study.

The statistical analysis, evaluated by GEE model, indicated that the DFP treated group had a significant increase over time of mean LVEF in comparison with the DFO treated group (Coefficient 0.97, 95\% Cl $0.37 ; 1.6, p=0.002$, Table 2 and Fig. 1 ). No statistically significant variation was found for the DFO-group over time (coefficient -0.43 , $95 \% \mathrm{CI}(-1.2 ; 0.32), p=0.26$, Table 2 and Fig. 1$)$.

The coefficient of the treatment-effect, indicating the difference between treatments, showed that there was not a statistically significant difference of LVEF between the two treated groups (coefficient -0.96 , $95 \% \mathrm{CI}-3.6 ; 1.7), p=0.47$, Table 2 and Fig. 1 ). Table 3 showed that in the patients with a LVEF less than $55 \%$, half ( 3 of 6 ) of DFP-treated group had an improved LVEF at the end of the study. Indeed, no

Table 2

GEE model to evaluate changes in mean ejection fraction levels in deferiprone (DFP)-group versus deferoximine (DFO)-group over time.

\begin{tabular}{lllc}
\hline & Coefficients $\left(\mathrm{SE}^{*}\right)$ & $95 \% \mathrm{Cl}^{\mathrm{a}}$ & $p$-Value \\
\hline Intercept & $58(1)$ & $(56-60)$ & $<0.0001$ \\
Treatment $^{\mathrm{c}}$ & $-0.96(1)$ & $(-4.0 ; 2.0)$ & 0.47 \\
Time $^{\mathrm{c}}$ & $0.97(0,31)$ & $(0.37 ; 1.6)$ & $<0.01$ \\
Treatment $^{\mathrm{b}}$ Time $^{\mathrm{d}}$ & $-0.43(0,38)$ & $(-1.0 ; 32)$ & 0.26 \\
\hline
\end{tabular}

a $\mathrm{SE}=$ standard error, $\mathrm{CI}=$ confidence interval.

b $p$-Value from null hypothesis coefficient $=0$ by Wald's test.

c Treatment is the variable indicating if a patient belongs to DFP-group or DFO-group; time is the variable indicating the consecutive years of observation, corresponding to $t=1,2,3,4$.

d Treatment $\times$ Time is the interaction effect between Treatment and Time. 


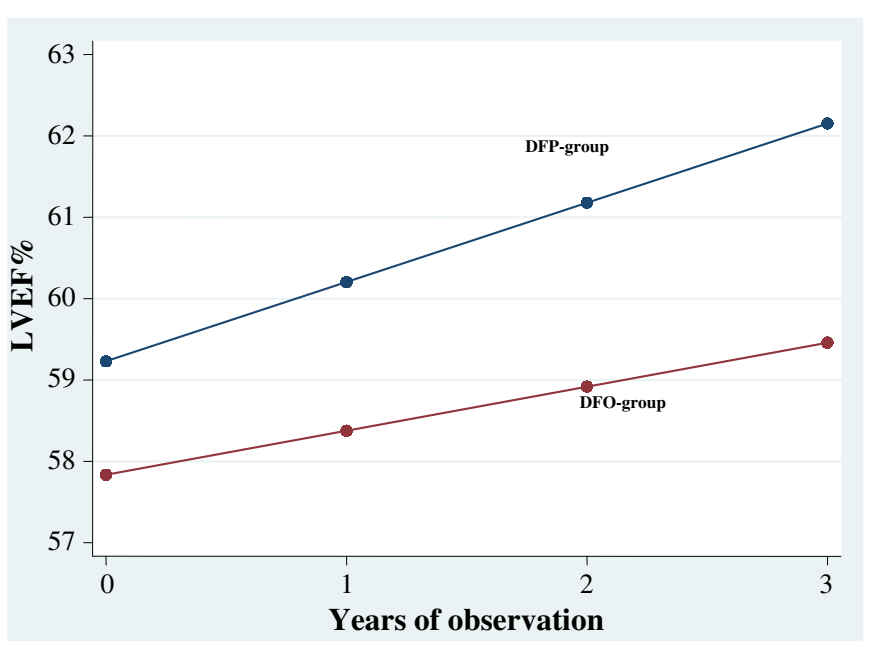

Fig. 1. Estimated profiles from the fitted generalized estimating equations (GEE) model for the deferiprone (DFP)-group and deferoxamine (DFO)-group. The baseline left ventricular ejection fraction (LVEF) for DFO group is $58 \pm 10 \%$ and for the DFP group is $59 \pm 4 \%$.

substantial difference was evident in the cohort included in the LVEF greater than $55 \%$ group (Table 3 ).

\section{Discussion}

The improvement of the LVEF after 1-year of DFP treatment as compared to DFO treatment has been described $[9,10]$. However, no comparison of LVEF in patients treated long-term with DFP compared to DFO has been reported.

Three retrospective studies and one-year prospective randomized trial have confirmed the superiority of DFP compared with DFO at removing cardiac iron as assessed by T2* MRI [15-18].

Moreover, different retrospective studies, in patients with thalassemia major, found that DFP was superior in comparison to DFO treatment in preventing cardiac death [19-21]. Whereas no cardiac deaths and no new cardiac events occurred in the DFP treated group, 10 cardiac deaths and 42 non-fatal cardiac events occurred in the DFO treated group [19]. This effect was not related to the serum ferritin levels [19]. The greater improvement of LVEF over time, obtained in the long-term DFP-treated group reported in this paper, may explain the results obtained in these previous clinical studies.

Moreover, similar findings have been described assessing retrospectively the variation of LVEF during the multicenter randomized, open-label, long-term, sequential DFP-DFO treatment compared to DFP-alone [8]. In this study, 99 patients underwent long-term echocardiography evaluation of LVEF, measured at baseline and every

Table 3

Yearly LVEF outcome in both chelation groups divided into $<55$ or $\geq 55 \%$ ejection fraction.

\begin{tabular}{|c|c|c|c|c|}
\hline & \multicolumn{4}{|c|}{ LVEF < $55 \%$} \\
\hline & \multicolumn{4}{|l|}{ Years } \\
\hline & 0 & 1 & 2 & 3 \\
\hline DFO no. (\%) & $26(24)$ & $17(16)$ & $22(20)$ & $20(18)$ \\
\hline \multirow[t]{4}{*}{ DFP no. (\%) } & $6.0(10)$ & $4.0(7.0)$ & $5.0(8.0)$ & $3.0(5.0)$ \\
\hline & \multicolumn{4}{|c|}{ LVEF > 55\% } \\
\hline & \multicolumn{4}{|l|}{ Years } \\
\hline & 0 & 1 & 2 & 3 \\
\hline DFO no. (\%) & $82(76)$ & $91(84)$ & $86(80)$ & $88(81)$ \\
\hline DFP no. (\%) & $54(90)$ & $56(93)$ & $55(92)$ & 57 (95) \\
\hline
\end{tabular}

DFO, deferoximine; DFP, deferiprone; LVEF, left ventricular ejection fraction.
12 months over three consecutive years. Among these, 39 and 60 received sequential DFP (75 mg/kg for 4 days/week)-DFO ( $50 \mathrm{mg} / \mathrm{kg}$ for 3 days/week) treatment or DFP treatment ( $75 \mathrm{mg} / \mathrm{kg}$ for 7 days/week). The paper concluded that DFP treatment was able to enhance LVEF, as determined by echocardiography, over time in comparison with sequential DFP-DFO [8].

A small increase in LVEF during one-year of chelation treatment among thalassemia major patients might be expected to reduce the risk of heart failure [22]. Changes in $\operatorname{LVEF}$ (3.1\% vs. 2.6\%), measured by CMR-T2*, during DFP or combined DFP-DFO treatment were associated with a risk reduction for the development of heart failure over 12 months (46\% vs. 26\%, respectively) [22]. Therefore, relatively small changes in LVEF may assume clinical importance for patients with thalassemia major.

A decrease in mean LVEF $\geq 7 \%$, as determined by 2-D echocardiography, was a strong predictive tool for identifying thalassemia patients at risk for death from heart failure in a cohort of 188 patients with thalassemia major who had serial observations of LVEF measurements for at least four consecutive years [23]. The paper reported the risk of death due to cardiac failure in patients with a mean reduction of LVEF $>7 \%$, was 4.93 with a $95 \%$ confidence interval of 1.6 to 15.0 $(p=0.005)$ [23]. The median time from the last echocardiography to death was 3 years. The ROC curve to detect a decrease in LVEF $\geq 7 \%$ showed high sensitivity (85\%) in predicting increased risk of death [23]. Kaplan-Meier survival curves, in thalassemia major patients with decreasing $\mathrm{LVEF}>7 \%$, was associated with a significant reduction in the probability of survival ( $p=0.0022)$ [23].

Iron-mediated damage to the heart, as reflected in LVEF function, may occur in heart cell mitochondria [24]. The heart may be particularly sensitive to iron-induced mitochondrial damage relative to other tissues because of the large number of mitochondria that are required for its high oxygen utilization and its low level of antioxidants [25]. Mitochondria generate ATP as a result of the action of respiratory chain complexes [26]. Since heart contractility could be down-regulated in response to the reduced ATP production due to iron-mediated mitochondrial damage, the high metabolic demands for increased cardiac muscle contraction would depend on the ability to restore mitochondrial activities and thereby improve myocardial cell function [22].

DFP, because of its lower molecular weight, may cross cell membranes more effectively and chelate iron from intracellular compartments. Therefore, the drug could protect heart mitochondria and thereby myocardial cell function [27]. A cardio-protective effect of DFP on mitochondrial function in cultured, iron-loaded heart cells has been reported, even at concentrations below the iron-mobilizing effect [28].

However, although these are the most likely hypothesis, the exact mechanisms remain to be so far fully elucidated.

In conclusion, this retrospective survey indicates that the long-term administration of DFP significantly enhances LVEF over time in comparison with DFO.

\section{Author's contributions}

A.F. and A.V. were the principal investigators and take primary responsibility for this paper. A.F., A.V. and A.M. wrote the paper. A.V. performed the statistical analysis. P.R., G.C., R.B., M.C. criticized and improved the paper. The remaining authors are responsible of the patients' clinical management.

\section{Conflict of interest}

The authors report no potential conflicts of interest.

\section{Acknowledgments}

The support of Piera Cutino Foundation was greatly appreciated. The patients' participation to the study is even respected. 


\section{References}

[1] D.R. Higgs, J.D. Engel, G. Stamatoyannopoulos, Thalassemia, Lancet (2012) 373-383.

[2] B. Modell, M. Khan, M. Darlison, Survival in $\beta$-thalassaemia major in the UK: data from the UK thalassaemia register, Lancet 355 (2000) 2051-2052.

[3] C. Borgna-Pignatti, S. Rugolotto, S.P. De Stefano, H. Zhao, M.D., G.C. Del Vecchio, M.A. Romeo, G.L. Forni, M.R. Gamberini, R. Ghilardi, A. Piga, A. Cnaan, Survival and complications in patients with thalassemia major treated with transfusion and deferoxamine, Haematologica 89 (10) (2004) 1187-1193.

[4] L.J. Anderson, S. Holden, B. Davis, E. Prescott, C.C. Charrier, N.H. Bunce, D.N. Firmin, B. Wonke, J. Porter, J.M. Walker, D.J. Pennell, Cardiovascular T2-star (T2*) magnetic resonance for the early diagnosis of myocardial iron overload, Eur. Heart J. 22 (2001) 2171-2179.

[5] B. Modell, M. Khan, M. Darlison, M.A. Westwood, D. Ingram, D.J. Pennell, Improved survival of thalassaemia major in the UK and relation to $\mathrm{T} 2 *$ cardiovascular magnetic resonance, J. Cardiovasc. Magn. Reson. 10 (Sep 25 2008) 42.

[6] M.J. Cunningham, E.A. Macklin, E.J. Neufeld, A.R. Cohen, Complications of beta-thalassemia major in North America, Blood 104 (1) (2004) 34-39.

[7] C. Borgna-Pignatti, M.D. Cappellini, P. De Stefano, G.C. Del Vecchio, G.L. Forni, M.R. Gamberini, R. Ghilardi, A. Piga, M.A. Romeo, H. Zhao, A. Cnaan, Cardiac morbidity and mortality in deferoxamine- or deferiprone-treated patients with thalassemia major, Blood 107 (9) (2006) 3733-3737, (2006).

[8] A. Maggio, A. Vitrano, G. Lucania, M. Capra, L. Cuccia, F. Gagliardotto, L. Pitrolo, L. Prossomariti, A. Filosa, V. Caruso, C. Gerardi, S. Campisi, P. Cianciulli, M. Rizzo, G. D'Ascola, A. Ciancio, R. Di Maggio, G. Calvaruso, G.R. Pantalone, P. Rigano, Long-term use of deferiprone significantly enhances left-ventricular ejection function in thalassemia major patients, Am. J. Hematol. 87 (2012) 732-733.

[9] A. Pepe, M. Lombardi, V. Positano, E. Cracolici, M. Capra, R. Malizia, L. Prossomariti, D. De Marchi, M. Midiri, A. Maggio, Evaluation of the efficacy of oral deferiprone in beta-thalassemia major by multislice multiecho T2*, Eur. J. Haematol. 76 (3) (2006) 183-192.

[10] D.J. Pennell, V. Berdoukas, M. Karagiorga, V. Ladis, A. Piga, A. Aessopos, E.D. Gotsis, M.A. Tanner, M.A.G.C. Smith, B. Wonke Westwood, R. Galanello, Randomized controlled trial of deferiprone or deferoxamine in beta-thalassemia major patients with asymptomatic myocardial siderosis, Blood 107 (9) (2006) 3738-3744.

[11] N.B. Schiller, P.M. Shah, M. Crawford, A. De Maria, R. Devereux, H. Feigenbaum, H. Gutgesell, N. Reichek, D. Sahn, I. Schnitteger, Recommendations for quantitation of the left ventricle by two-dimensional echocardiography. American Society of Echocardiography Committee on Standards. Subcommittee on quantitation of Two-Dimensional Echocardiograms, J. Am. Soc. Echocardiogr. 2 (5) (1989) 358-367.

[12] T. Cogliandro, G. Derchi, L. Mancuso, B. Pannone, A. Pepe, M. Pili, P. Bina, P. Cianciulli, V. De Sanctis, A. Maggio, Guideline recommendations for heart complications in thalassemia major, J. Cardiovasc. Med. (Hagerstown) 9 (2008) 515-525.

[13] V. Di Marco, M. Capra, E. Angelucci, C. Borgna-Pignatti, P. Telfer, P. Harmatz, A. Kattamis, L. Prossamariti, A. Filosa, D. Rund, M.R. Gamberini, P. Cianciulli, M. De Montalembert, F. Gagliardotto, G. Foster, J.D. Grangè, F. Cassarà, A. Iacono, M.D. Cappellini, G.M. Brittenham, D. Prati, A. Pietrangelo, A. Craxì, A. Maggio, Italian Society for the Study of Thalassemia an Haemoglobinopathies; Italian Association for the Study of the Liver, Management of chronic viral hepatitis in patients with thalassemia: recommendations from an international panel, Blood 116 (16) (2010) 2875-2883, (Epub).

[14] D. Hedeker, R.D. Gibbons, Longitudinal Data Analysis, Hoboken Wiley, New Jersey, 2006.

[15] L.J. Anderson, B. Wonke, E. Prescott, S. Holden, J.M. Walker, D.J. Pennell, Comparison of effects of oral deferiprone and subcutaneous desferrioxamine on myocardial iron concentrations and ventricular function in beta-thalassemia, Lancet 360 (2002) $516-520$.

[16] A. Maggio, G. D'Amico, A. Morabito, M. Capra, C. Ciaccio, P. Cianciulli, F. Di Gregorio, G. Garozzo, R. Malizia, C. Magnani, G. Quarta, M. Rizzo, D.G. D'Ascola, A. Rizzo, M. Midiri, Deferiprone versus deferoxamine in patients with thalassemia major: a randomized clinical trial, Blood Cell Mol. Dis. 28 (2) (2002) 196-208.

[17] A. Pepe, A. Meloni, M. Capra, P. Cianciulli, L. Prossomariti, C. Malaventura, M.C. Putti, A. Lippi, M.A. Romeo, M.G. Bisconte, A. Filosa, V. Caruso, A. Quarta, L. Pitrolo, M. Missere, M. Midiri, G. Rossi, V. Positono, M. Lombardi, A. Maggio, Deferasirox, deferiprone and desferrioxamine treatment in thalassemia major patients: cardiac iron and function comparison determined by quantitative magnetic resonance imaging, Haematologica 96 (1) (2011) 41-47.

[18] D.J. Pennell, V. Berdoukas, M. Karagiorgia, V. Ladis, A. Piga, A. Aessopos, E.D. Gotsis, M.A. Tanner, G.C. Smith, M.A. Westwood, B. Wonke, R. Galanello, Randomized controlled trial deferiprone or deferoxamine in beta-thalassemia major patients with asymptomatic myocardial siderosis, Blood 107 (9) (2006) 3738-3744

[19] C. Borgna-Pignatti, M.D. Cappellini, P. De Stefano, G.C. Del Vecchio, G.L. Forni, M.R Gamberini, R. Ghilardi, A. Piga, M.A. Romeo, H. Zhao, A. Cnaan, Cardiac morbidity and mortality in deferoxamine or deferiprone treated patients with thalassemia major, Blood 107 (9) (2006) 3733-3737, (P).

[20] A. Piga, C. Gaglioti, E. Fogliacco, F. Tricta, Comparative effects of deferiprone and deferoxamine on survival and cardiac disease in patients with thalassemia major: a retrospective analysis, Haematologica 88 (5) (2003) 489-496.

[21] A. Maggio, A. Vitrano, M. Capra, L. Cuccia, F. Gagliardotto, A. Filosa, C. Magnano, M Rizzo, V. Caruso, C. Gerardi, C. Argento, S. Campisi, F. Cantella, F. Commendatore, D.G. D'Ascola, C. Fidone, A. Ciancio, M.C. Galati, G. Giuffrida, G. Giugno, T. Lombardo, L. Prossomariti, R. Malizia, A. Meo, G. Roccamo, M.A. Romeo, P. Violi, P. Cianciulli, P. Rigano, Improving survival with deferiprone treatment in patients with thalassemia major: a prospective multicenter randomized clinical trial under the auspices of the Italian Society for Thalassemia and Hemoglobinopathies, Blood Cells Mol. Dis. 42 (3) (2009) 247-251.

[22] D.J. Pennell, J.P. Carpenter, M. Roughton, Z. Cabantchik, On improvement in ejection fraction with iron chelation in thalassemia major and the risk of future heart failure, J. Cardiovasc. Magn. Reson. 13 (2011) 45-53.

[23] A. Maggio, A. Vitrano, G. Calvaruso, R. Barone, P. Rigano, L. Mancuso, L. Cuccia, M. Capra, L. Pitrolo, L. Prossomariti, A. Filosa, V. Caruso, C. Gerardi, S. Campisi, P. Cianciulli, A. Elfteriou, M. Angastiniotis, H. Hamza, P. Telfer, J.M. Walker, A. Phrommintikul, N. Chattipakorn, Serial echocardiographic left ventricular ejection fraction measurements: a tool for detecting thalassemia major patients at risk of cardiac death, Blood Cells Mol. Dis. 50 (4) (2013) 241-246, (Epub).

[24] X. Gao, M. Qian, J.L. Campian, J. Marshall, Z. Zhou, A.M. Roberts, Y.J. Kang, S.D. Prabhu, X.F. Sun, J.W. Eaton, Mitochondrial dysfunction may explain the cardiomyopathy of chronic iron overload, Free Radic. Biol. Med. 49 (2010) 401-407.

[25] E.J. Lesnefsky, S. Moghaddas, B. Tandler, J. Kerner, C.L. Hoppel, Mitochondrial dysfunction in cardiac disease: ischemia-reperfusion, aging, and heart failure, J. Mol. Cell. Cardiol. 33 (2001) 1065-1089.

[26] G. Link, A. Saada, A. Pinson, A.M. Konijn, C. Hershko, Mitochondrial respiratory enzymes are a major target of iron toxicity in rat heart cells, J. Lab. Clin. Med. 131 (1998) 466-474.

[27] H. Glickstein, R.B. El, M. Shvartsman, Z.L. Cabantchik, Intracellular labile iron pools as direct targets of iron chelators: a fluorescence study of chelator action in living cells, Blood 106 (9) (2005) 3242-3250.

[28] G. Link, A.M. Konijn, C. Hershko, Cardioprotective effect of alpha-tocopherol, ascorbate, deferoxamine, and deferiprone: mitochondrial function in cultured, iron-loaded heart cells, J. Lab. Clin. Med. 133 (2) (1999) 179-188. 